\title{
Superconductivity, Antiferromagnetism, and Kinetic Correlation in Strongly Correlated Electron Systems
}

\author{
Takashi Yanagisawa \\ Electronics and Photonics Research Institute, National Institute of Advanced Industrial Science and Technology, \\ 1-1-1 Umezono, Tsukuba, Ibaraki 305-8568, Japan
}

Correspondence should be addressed to Takashi Yanagisawa; t-yanagisawa@aist.go.jp

Received 18 August 2015; Accepted 28 September 2015

Academic Editor: Artur P. Durajski

Copyright (C) 2015 Takashi Yanagisawa. This is an open access article distributed under the Creative Commons Attribution License, which permits unrestricted use, distribution, and reproduction in any medium, provided the original work is properly cited.

\begin{abstract}
We investigate the ground state of two-dimensional Hubbard model on the basis of the variational Monte Carlo method. We use wave functions that include kinetic correlation and doublon-holon correlation beyond the Gutzwiller ansatz. It is still not clear whether the Hubbard model accounts for high-temperature superconductivity. The antiferromagnetic correlation plays a key role in the study of pairing mechanism because the superconductive phase exists usually close to the antiferromagnetic phase. We investigate the stability of the antiferromagnetic state when holes are doped as a function of the Coulomb repulsion $U$. We show that the antiferromagnetic correlation is suppressed as $U$ is increased exceeding the bandwidth. High-temperature superconductivity is possible in this region with enhanced antiferromagnetic spin fluctuation and pairing interaction.
\end{abstract}

\section{Introduction}

The study of high-temperature superconductivity has attracted much attention since the discovery of cuprate high-temperature superconductors [1]. It is very important to clarify the properties of electronic states in the $\mathrm{CuO}_{2}$ plane [2-9]. The model for the $\mathrm{CuO}_{2}$ plane is called the $\mathrm{d}-\mathrm{p}$ model (or is called the three-band Hubbard model). We often consider the simplified model, by neglecting oxygen sites in the $\mathrm{CuO}_{2}$ plane, called the (single-band) Hubbard model [10-15].

It remains unresolved as to whether the two-dimensional Hubbard model has a superconducting phase or not $[16,17]$. It is believed that the electron correlation between electrons plays a significant role in cuprate superconductors. It is obvious that interaction with large energy scale is responsible for realization of high-temperature superconductivity. This subject has been investigated for more than two decades by using electronic models such as the two-dimensional Hubbard model, the d-p model, and the ladder Hubbard model [18-21].

The antiferromagnetic (AF) correlation plays a primarily important role in correlated electron systems. For example, the existence of striped states [22-29] can be understood on the basis of the two-dimensional Hubbard model $[9,30,31]$.
The checkerboard-like density-wave modulation, observed by scanning tunneling microscopy (STM) [32-34], is also possible in some region of the parameter space in the Hubbard model [31]. The possibility of the coexistent state of antiferromagnetism and superconductivity has been reported $[35,36]$, and we can show the coexistence using the Hubbard model [9]. Thus the two-dimensional Hubbard model can describe some of anomalous properties reported for cuprate superconductors. The spin fluctuation, which is one of candidates of attractive interaction for high-temperature superconductivity, comes from the antiferromagnetic spin correlation. It is thus important to examine the stability of the antiferromagnetic state.

In the mean-field theory the antiferromagnetic correlation is enhanced as $U$ is increased. This is also the case for the wave function of simple Gutzwiller ansatz. When we consider electron correlation beyond the Gutzwiller ansatz (meanfield wave function), the nature of the antiferromagnetism changes in the strongly correlated region where $U$ is larger than the bandwidth. The AF correlation is increased as $U$ is increased from zero and is maximally enhanced when $U$ is about the bandwidth. The AF correlation shows a tendency to be suppressed when $U$ is further increased more than the bandwidth. This is because we must suppress AF correlation 
to get the kinetic energy gain to lower the ground state energy. This indicates that the spin fluctuation becomes large in the large- $U$ region.

In this paper we investigate the stability of AF ordered state using the wave functions that take account of kinetic correlation and doublon-holon correlation. We show the results obtained by the variational Monte Carlo method.

\section{Model and Wave Functions}

The Hubbard Hamiltonian is written as

$$
H=\sum_{i j \sigma} t_{i j} c_{i \sigma}^{\dagger} c_{j \sigma}+U \sum_{i} n_{i \uparrow} n_{i \downarrow}
$$

where $\left\{t_{i j}\right\}$ are transfer integrals and $U$ is the on-site Coulomb energy. The transfer integral $t_{i j}$ for nearest-neighbor pairs $\langle i j\rangle$ is denoted as $t_{i j}=-t$ and that for next-nearest-neighbor pair $\langle\langle i j\rangle\rangle$ is $t_{i j}=-t^{\prime}$. Otherwise, $t_{i j}$ vanishes. We denote the number of sites as $N$ and the number of electrons as $N_{e}$. The energy unit is given by $t$.

The simple wave function is given by the Gutzwiller function:

$$
\psi_{G}=P_{G} \psi_{0}
$$

where $P_{G}$ is the Gutzwiller operator

$$
P_{G}=\prod_{j}\left(1-(1-g) n_{j \uparrow} n_{j \downarrow}\right)
$$

with the parameter $g$ in the range of $0 \leq g \leq 1 . \psi_{0}$ is a trial function of the noninteracting state. To investigate the superconducting state, we use the BCS wave function $\psi_{\mathrm{BCS}}$ for $\psi_{0}$ with the gap parameter $\Delta$. The condensation energy is defined as

$$
E_{\text {cond }}=E(\Delta=0)-E\left(\Delta=\Delta_{\text {opt }}\right)
$$

for the optimized gap function $\Delta_{\text {opt }}$.

We take into account intersite correlation effects in the wave function by multiplying a correlation operator $e^{-\lambda K}$ :

$$
\psi_{\lambda}=e^{-\lambda K} \psi_{G}
$$

where $K$ indicates the kinetic term of the Hamiltonian $K=$ $\sum_{i j \sigma} t_{i j} c_{i \sigma}^{\dagger} c_{j \sigma}$ and $\lambda$ is a real constant which is the variational parameter to be optimized [37-39]. This wave function is a first approximation to the wave function used in quantum Monte Carlo method [40].

The other way to improve the wave function is to consider the doublon-holon correlation that is controlled by the operator given by [41]

$$
\begin{aligned}
P_{\mathrm{dh}} & =\prod_{j}(1 \\
& \left.-(1-\eta) \prod_{\tau}\left[d_{j}\left(1-e_{j+\tau}\right)+e_{j}\left(1-d_{j+\tau}\right)\right]\right) .
\end{aligned}
$$

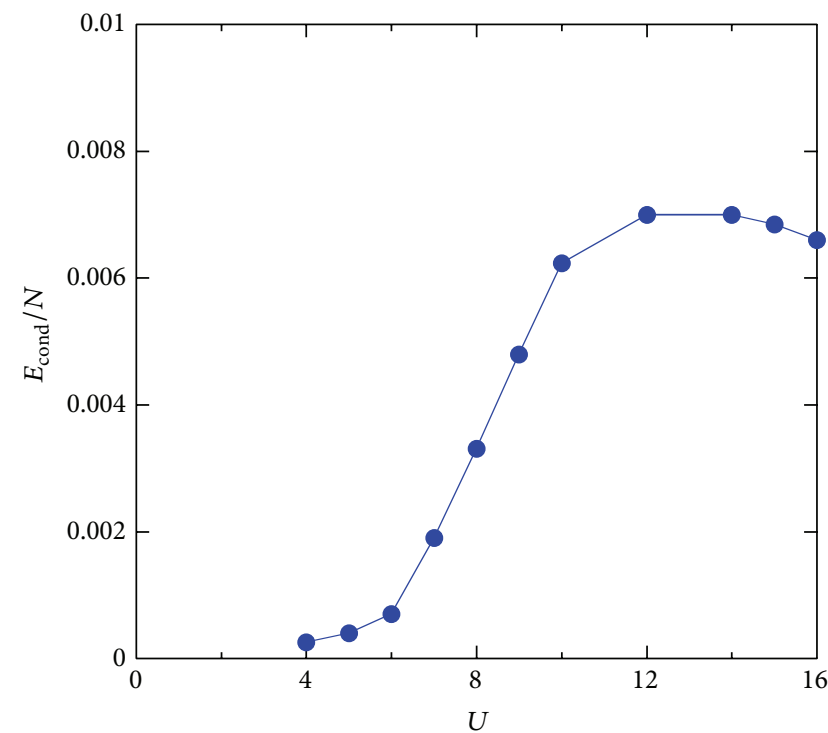

FIGURE 1: Superconducting condensation energy as a function of $U$ for the Gutzwiller function. The system is $10 \times 10$ lattice with $t^{\prime}=$ -0.2 and the electron number is $N_{e}=84$. We impose the periodic boundary condition in one direction and antiperiodic boundary condition in the other direction.

$d_{j}$ is the operator for the doubly occupied site given as $d_{j}=$ $n_{j \uparrow} n_{j \downarrow}$ and $e_{j}$ is that for the empty site given by $e_{j}=(1-$ $\left.n_{j \uparrow}\right)\left(1-n_{j \downarrow}\right) \cdot \eta$ is the variational parameter in the range of $0 \leq \eta \leq 1$. The doublon-holon wave function is written as

$$
\psi_{\eta}=P_{\mathrm{dh}} \psi_{\mathrm{G}}
$$

We evaluate physical quantities for the Gutzwiller function $\psi_{G}$ and optimized wave functions $\psi_{\lambda}$ and $\psi_{\eta}$ using the variational Monte Carlo method [42, 43].

\section{Antiferromagnetism and Superconductivity}

We first show the superconducting condensation energy and optimized superconducting gap function $\Delta$ as a function of $U$ in Figures 1 and 2, respectively. These results were obtained by using the Gutzwiller function with a BCS trial function. The results show that $\Delta$ increases as $U$ is increased and will have a maximum at some $U$. This indicates that the superconducting state becomes more stable in the strongly correlated region for larger $U$. The region with $U$ being greater than the bandwidth is called the strongly correlated region [41, 44]. It is difficult to observe a clear sign of superconductivity in weakly correlated region; for example, $U$ is less than $6 t$, by numerical calculations. This is consistent with the results obtained by quantum Monte Carlo methods [17, 39, 45].

This result suggests that there is some effect which induces an attractive interaction to form electron pairings in strongly correlated region. We examine the stability of AF ordered state in this region. For this purpose we employ the kinetic correlation function $\psi_{\lambda}=e^{-\lambda K} \psi_{G}$ and the doublon-holon correlation function $\psi_{\eta}=P_{\mathrm{dh}} \psi_{\mathrm{G}}$. We compare the ground state energy for $\psi_{G}, \psi_{\eta}$, and $\psi_{\lambda}$ in Table 1. Among these wave 


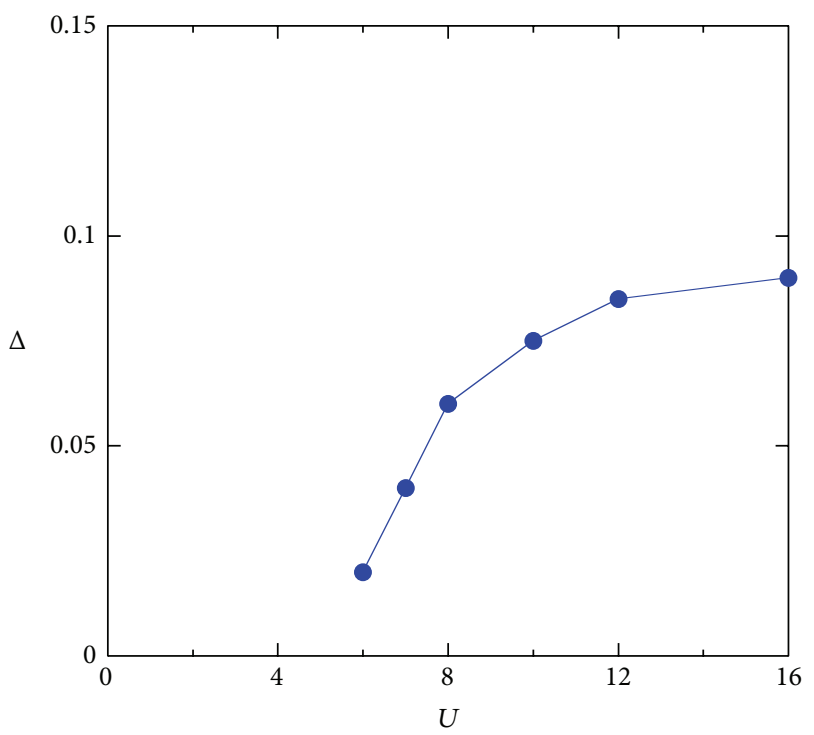

FIGURE 2: Optimized superconducting gap function as a function of $U$ for the BCS Gutzwiller function. The conditions are the same as in Figure 1.

TABLE 1: Ground state energy per site for wave functions $\psi_{G}$, doublon-holon function $\psi_{\eta}$ and $\psi_{\lambda}$. The parameters are $N_{e}=86$, $U=10, t^{\prime}=-0.2$ on $10 \times 10$ lattice with periodic boundary conditions. $\Delta_{\mathrm{AF}}$ is the antiferromagnetic order parameter.

\begin{tabular}{lccccc}
\hline Wave function & $E / N$ & $g$ & $\Delta_{\mathrm{AF}}$ & $\eta$ & $\lambda$ \\
\hline$\psi_{G}$ & -0.5859 & 0.21 & 0.0 & 1.0 & 0 \\
$\psi_{G}(\mathrm{AF})$ & -0.5953 & 0.21 & 0.2 & 1.0 & 0 \\
$\psi_{\eta}=P_{\mathrm{dh}} \psi_{G}$ & -0.6179 & 0.21 & 0.0 & 0.78 & 0 \\
$\psi_{\lambda}=e^{-\lambda K} \psi_{G}$ & -0.6643 & 0.06 & 0.0 & 1.0 & 0.06 \\
\hline
\end{tabular}

functions, $\psi_{\lambda}$ shows the lowest ground state energy. This indicates that the intersite correlation induced by the kinetic operator $K$ is important in the correlated region. In Figure 3 we show the kinetic energy which is the expectation value of the kinetic term as well as the ground state energy as a function of $\lambda$ for $\psi_{\lambda}$. The kinetic energy is lowered appreciably by the kinetic correlation operator $e^{-\lambda K}$.

When $U$ is large, there is a competition between antiferromagnetic energy gain and kinetic energy gain to lower the total energy. The AF energy gain, being proportional to the AF exchange coupling $J \propto t^{2} / U$, is reduced gradually as $U$ is increased. We show this in Figure 4 where the energy gain of the AF state with reference to the normal state is shown as a function of $U$. When $U$ is increased more than the bandwidth, the antiferromagnetism is suppressed and its energy gain is decreased as $U$ is increased. This indicates that there is a large AF spin fluctuation in this region. This shows a possibility of AF fluctuation induced electron pairing in the strongly correlated region.

\section{Summary}

We have investigated the two-dimensional Hubbard model by adopting the wave function that takes into account

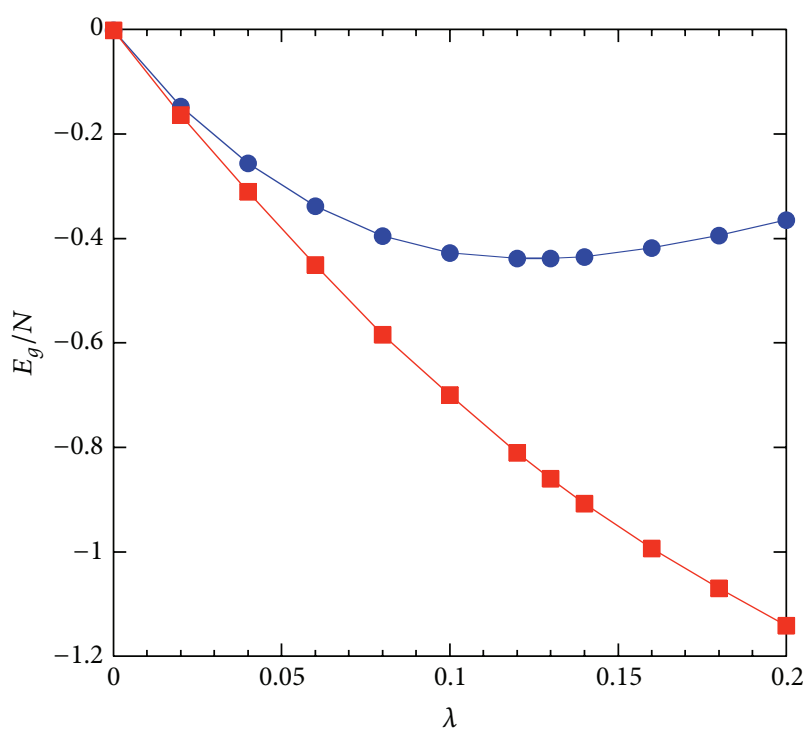

FIGURE 3: Ground state energy (circles) and the kinetic energy (squares) as a function of $\lambda$, evaluated using $\psi_{\lambda}$. The parameters are $U=10, t^{\prime}=-0.2$, and $N_{e}=100$.

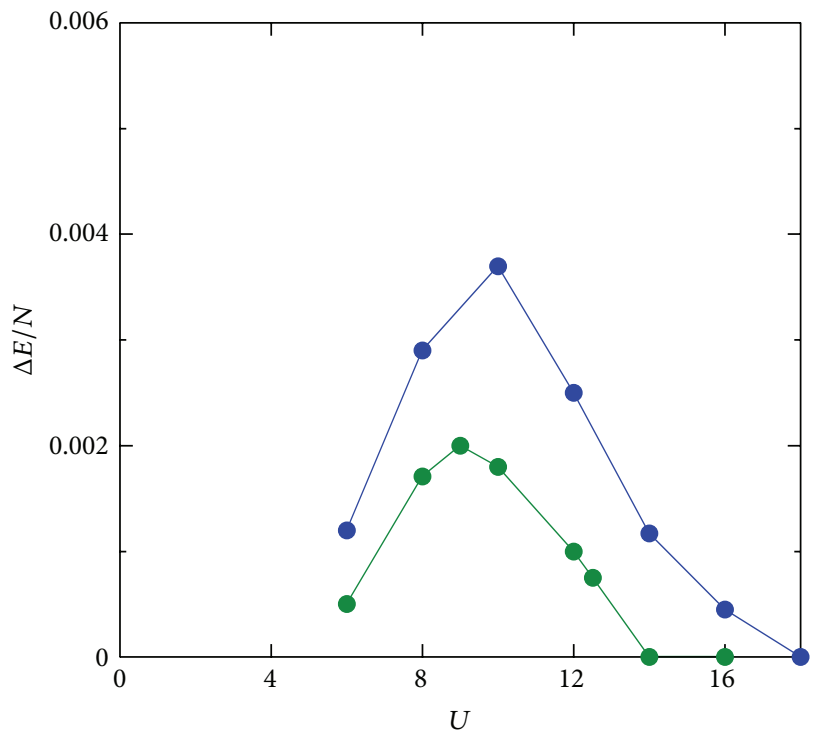

FIGURE 4: Antiferromagnetic condensation energy as a function of $U$. The system is $10 \times 10$ lattice with $t^{\prime}=-0.2$. The electron number is $N_{e}=86$ (lower curve) and $N_{e}=84$ (upper curve). In the latter $N_{e}=$ 84 case we have an open shell; that is, the highest occupied electron level is partially filled. We use the periodic boundary condition in both directions.

intersite electron correlation using the variational Monte Carlo method. The condensation energy becomes large in the strongly correlated region, suggesting a possibility of high-temperature superconductivity. The reduction of antiferromagnetic correlation suggests large spin fluctuation in this region. We expect that this spin fluctuation in strongly correlated region induces an attractive interaction which promotes superconductivity. In the weakly correlated region for $U / t$ less than about 6 , there is also spin fluctuation because 
the AF order is suppressed as $U$ is decreased. In numerical calculations, however, the pairing state is not stabilized in the weakly correlated region. This indicates that only the spin fluctuation effect cannot promote electron pairing and we further need strong correlation to account for hightemperature superconductivity.

It is also important to discuss the role of other parameters in the Hubbard model. In particular, the next-nearestneighbor transfer $t^{\prime}$ is important since the density of states crucially depends on $t^{\prime}$ due to the van Hove singularity of a two-dimensional system. The nearest-neighbor Coulomb interaction $V$ is also important. The on-site Coulomb interaction $U$ is renormalized by $V$ and $V$ will change the nature of antiferromagnetic correlation.

It is necessary to consider three-dimensional electronic models because real cuprate superconductors are threedimensional layered materials. It is important to investigate the electronic state of layered cuprates because an interlayer interaction may bring about interesting phenomena. They can be regarded as a multiband superconductor and have potential for new phenomena [46-52].

It is also necessary to examine the effect of strong correlation on the electron-phonon interaction [53-56]. There is a possibility that electron-phonon and electron-electron interactions cooperate to realize a high-temperature superconductor. The electron-phonon coupling may induce longrange attractive interaction. We expect that the long-range part of attractive interaction may cooperate with the on-site repulsive interaction to induce superconductivity.

\section{Conflict of Interests}

The author declares that there is no conflict of interests regarding the publication of this paper.

\section{Acknowledgments}

The author thanks K. Yamaji and M. Miyazaki for useful discussions. This work was supported in part by grant-inaid from the Ministry of Education, Culture, Sports, Science and Technology (MEXT) of Japan. A part of numerical calculations was performed at the Supercomputer Center of the Institute for Solid State Physics, University of Tokyo.

\section{References}

[1] K. H. Bennemann and J. B. Ketterson, Eds., The Physics of Superconductors, Volume I and Volume II, Springer, Berlin, Germany, 2003.

[2] V. J. Emery, "Theory of high- $T_{c}$ superconductivity in oxides," Physical Review Letters, vol. 58, no. 26, p. 2794, 1987.

[3] J. E. Hirsch, E. Loh Jr., D. J. Scalapino, and S. Tang, "Pairing interaction in CuO clusters," Physical Review B, vol. 39, no. 1, article 243, 1989.

[4] R. T. Scalettar, D. J. Scalapino, R. L. Sugar, and S. R. White, "Antiferromagnetic, charge-transfer, and pairing correlations in the three-band Hubbard model," Physical Review B, vol. 44, no. 2, pp. 770-781, 1991.
[5] C. Weber, A. Läuchli, F. Mila, and T. Giamarchi, "Orbital currents in extended hubbard models of high- $T_{c}$ cuprate superconductors," Physical Review Letters, vol. 102, no. 1, Article ID 017005, 2009.

[6] B. Lau, M. Berciu, and G. A. Sawatzky, "High-spin polaron in lightly doped $\mathrm{CuO}_{2}$ planes," Physical Review Letters, vol. 106, no. 3, Article ID 036401, 2011.

[7] T. Yanagisawa, S. Koike, and K. Yamaji, "Ground state of the three-band Hubbard model," Physical Review B, vol. 64, no. 18, Article ID 184509, 2001.

[8] T. Yanagisawa, M. Miyazaki, S. Koikegami, S. Koike, and K. Yamaji, "Lattice distortions, incommensurability, and stripes in the electronic model for high- $T_{c}$ cuprates," Physical Review B, vol. 67, no. 13, Article ID 132408, 2003.

[9] T. Yanagisawa, M. Miyazaki, and K. Yamaji, "Incommensurate antiferromagnetism coexisting with superconductivity in twodimensional d-p model," Journal of the Physical Society of Japan, vol. 78, no. 1, Article ID 013706, 2009.

[10] J. Hubbard, "Electron correlations in narrow energy bands," Proceedings of the Royal Society of London Series A: Mathematical and Physical, vol. 276, no. 1365, pp. 238-257, 1963.

[11] J. E. Hirsch, "Two-dimensional Hubbard model: numerical simulation study," Physical Review B, vol. 31, no. 7, pp. 44034419, 1985.

[12] H. Yokoyama and H. Shiba, "Variational Monte-Carlo studies of superconductivity in strongly correlated electron systems," Journal of the Physical Society of Japan, vol. 57, no. 7, pp. 24822493, 1988.

[13] T. Yanagisawa and Y. Shimoi, "Exact results in strongly correlated electrons-spin-reflection positivity and the perronfrobenius theorem," International Journal of Modern Physics B, vol. 10, no. 25, pp. 3383-3450, 1996.

[14] K. Yamaji, T. Yanagisawa, and S. Koike, "Bulk limit of superconducting condensation energy in 2D Hubbard model," Physica B: Condensed Matter, vol. 284-288, part 1, pp. 415-416, 2000.

[15] T. Yanagisawa, "Kosterlitz-thouless transition in two-dimensional hubbard model evidenced from quantum monte carlo calculations of susceptibilities," Journal of the Physical Society of Japan, vol. 79, no. 6, Article ID 063708, 4 pages, 2010.

[16] N. Bulut, " $d_{x^{2}-y^{2}}$ superconductivity and the Hubbard model," Advanced in Physics, vol. 51, no. 7, pp. 1587-1667, 2002.

[17] T. Aimi and M. Imada, "Does simple two-dimensional hubbard model account for high- $T_{c}$ superconductivity in copper oxides?" Journal of the Physical Society of Japan, vol. 76, no. 11, Article ID 113708, 2007.

[18] R. M. Noack, N. Bulut, D. J. Scalapino, and M. G. Zacher, "Enhanced $d_{x^{2}-y^{2}}$ pairing correlations in the two-leg Hubbard ladder," Physical Review B, vol. 56, no. 12, article 7162, 1997.

[19] S. Koike, K. Yamaji, and T. Yanagisawa, "Effect of the mediumrange transfer energies to the superconductivity in the twochain Hubbard model," Journal of the Physical Society of Japan, vol. 68, no. 5, pp. 1657-1663, 1999.

[20] K. Yamaji, Y. Shimoi, and T. Yanagisawa, "Superconductivity indications of the two-chain Hubbard model due to the twoband effect," Physica C: Superconductivity and its applications, vol. 235-240, no. 4, pp. 2221-2222, 1994.

[21] T. Yanagisawa, Y. Shimoi, and K. Yamaji, "Superconducting phase of a two-chain Hubbard model," Physical Review B, vol. 52, no. 6, pp. R3860-R3863, 1995.

[22] J. M. Tranquada, J. D. Axe, N. Ichikawa, Y. Nakamura, S. Uchida, and B. Nachumi, "Neutron-scattering study of stripe-phase 
order of holes and spins in $\mathrm{La}_{1.48} \mathrm{Nd}_{0.4} \mathrm{Sr}_{0.12} \mathrm{CuO}_{4}$," Physical Review B, vol. 54, no. 10, pp. 7489-7499, 1996.

[23] T. Suzuki, T. Goto, K. Chiba et al., "Observation of modulated magnetic long-range order in $\mathrm{La}_{1.88} \mathrm{Sr}_{0.12} \mathrm{CuO}_{4}$," Physical Review $B$, vol. 57, no. 6, Article ID R3229, 1998.

[24] K. Yamada, C. H. Lee, K. Kurahashi et al., "Doping dependence of the spatially modulated dynamical spin correlations and the superconducting-transition temperature in $\mathrm{La}_{2-x} \mathrm{Sr}_{x} \mathrm{CuO}_{4}$," Physical Review B, vol. 57, no. 10, pp. 6165-6172, 1998.

[25] M. Arai, T. Nishijima, Y. Endoh et al., "Incommensurate spin dynamics of underdoped superconductor $\mathrm{YBa}_{2} \mathrm{Cu}_{3} \mathrm{O}_{6.7}$," Physical Review Letters, vol. 83, no. 3, pp. 608-611, 1999.

[26] H. A. Mook, P. Dai, F. Doğan, and R. D. Hunt, "One-dimensional nature of the magnetic fluctuations in $\mathrm{YBa}_{2} \mathrm{Cu}_{3} \mathrm{O}_{6.6}$," Nature, vol. 404, no. 6779, pp. 729-731, 2000.

[27] S. Wakimoto, R. J. Birgeneau, M. A. Kastner et al., "Direct observation of a one-dimensional static spin modulation in insulating $\mathrm{La}_{1.95} \mathrm{Sr}_{0.05} \mathrm{Cuo}_{4}$," Physical Review B, vol. 61, article $3699,2000$.

[28] A. Bianconi, N. L. Saini, A. Lanzara et al., "Determination of the local lattice distortions in the $\mathrm{CuO}_{2}$ plane of $\mathrm{La}_{1.85} \mathrm{Sr}_{0.15} \mathrm{CuO}_{4}$," Physical Review Letters, vol. 76, no. 18, pp. 3412-3415, 1996.

[29] A. Bianconi, "Quantum materials: Shape resonances in superstripes," Nature Physics, vol. 9, no. 9, pp. 536-537, 2013.

[30] M. Miyazaki, T. Yanagisawa, and K. Yamaji, "Diagonal stripe states in the light-doping region in the two-dimensional Hubbard model," Journal of the Physical Society of Japan, vol. 73, no. 7, pp. 1643-1646, 2004.

[31] M. Miyazaki, K. Yamaji, T. Yanagisawa, and R. Kadono, "Checkerboard states in the two-dimensional hubbard model with the Bi2212-type band," Journal of the Physical Society of Japan, vol. 78, no. 4, Article ID 043706, 4 pages, 2009.

[32] J. E. Hoffman, E. W. Hudson, K. M. Lang et al., "A four unit cell periodic pattern of quasi-particle states surrounding Vortex cores in $\mathrm{Bi}_{2} \mathrm{Sr}_{2} \mathrm{CaCu}_{2} \mathrm{O}_{8+\delta}$, Science, vol. 295, no. 5554, pp. 466469, 2002.

[33] W. D. Wise, M. C. Boyer, K. Chatterjee et al., "Charge-densitywave origin of cuprate checkerboard visualized by scanning tunnelling microscopy," Nature Physics, vol. 4, pp. 696-699, 2008.

[34] T. Hanaguri, C. Lupien, Y. Kohsaka et al., "A 'checkerboard' electronic crystal state in lightly hole-doped $\mathrm{Ca}_{2-x} \mathrm{Na}_{x} \mathrm{CuO}_{2} \mathrm{Cl}_{2}$," Nature, vol. 430, no. 7003, pp. 1001-1005, 2004.

[35] H. Mukuda, M. Abe, Y. Araki et al., "Uniform mixing of high $-T_{c}$ superconductivity and antiferromagnetism on a single $\mathrm{CuO}_{2}$ plane of a Hg-based five-layered cuprate," Physical Review Letters, vol. 96, no. 8, Article ID 087001, 2006.

[36] A. Crisan, Y. Tanaka, A. Iyo et al., "Coexistence of superconductivity and antiferromagnetism in $\mathrm{HgBa}_{2} \mathrm{Ca}_{4} \mathrm{Cu}_{5} \mathrm{O}_{y}$ : multiharmonic susceptibility and vortex dynamics study," Physical Review B, vol. 76, no. 21, Article ID 212508, 4 pages, 2007.

[37] T. Yanagisawa, S. Koike, and K. Yamaji, "Off-diagonal wave function monte carlo studies of hubbard nodel I," Journal of the Physical Society of Japan, vol. 67, no. 11, pp. 3867-3874, 1998.

[38] T. Yanagisawa, S. Koike, and K. Yamaji, " $d$-Wave state with multiplicative correlation factors for the Hubbard model," Journal of the Physical Society of Japan, vol. 68, no. 11, pp. 36083614, 1999.

[39] T. Yanagisawa, M. Miyazaki, and K. Yamaji, "Correlatedelectron systems and high-temperature superconductivity," Journal of Modern Physics, vol. 4, pp. 33-64, 2013.
[40] T. Yanagisawa, "Enhanced pair correlation functions in the twodimensional Hubbard model," New Journal of Physics, vol. 15, no. 3, Article ID 033012, 2013.

[41] H. Yokoyama, M. Ogata, and Y. Tanaka, "Mott transitions and $d$ wave superconductivity in half-filled Hubbard model on square lattice with geometric frustration," Journal of the Physical Society of Japan, vol. 75, no. 11, Article ID 114706, 2006.

[42] T. Nakanishi, K. Yamaji, and T. Yanagisawa, "Variational Monte Carlo indications of $d$-wave superconductivity in the twodimensional Hubbard model," Journal of the Physical Society of Japan, vol. 66, no. 2, pp. 294-297, 1997.

[43] K. Yamaji, T. Yanagisawa, T. Nakanishi, and S. Koike, "Variational Monte Carlo study on the superconductivity in the twodimensional Hubbard model," Physica C: Superconductivity, vol. 304, no. 3-4, pp. 225-238, 1998.

[44] T. Yanagisawa and M. Miyazaki, "Mott transition in cuprate high-temperature superconductors," EPL, vol. 107, no. 2, Article ID 27004, 2014.

[45] S. Zhang, J. Carlson, and J. E. Gubernatis, "Constrained path Monte Carlo method for fermion ground states," Physical Review B-Condensed Matter and Materials Physics, vol. 55, no. 12, article 7464, 1997.

[46] Y. Tanaka, A. Iyo, S. Itoh, K. Tokiwa, T. Nishio, and T. Yanagisawa, "Experimental observation of a possible first-order phase transition below the superconducting transition temperature in the multilayer cuprate superconductor $\mathrm{HgBa}_{2} \mathrm{Ca}_{4} \mathrm{Cu}_{5} \mathrm{O}_{y}$," Journal of the Physical Society of Japan, vol. 83, no. 7, Article ID 074705, 2014.

[47] V. Stanev and Z. Tesanovic, "Three-band superconductivity and the order parameter that breaks time-reversal symmetry," Physical Review B, vol. 81, Article ID 134522, 2010.

[48] Y. Tanaka and T. Yanagisawa, "Chiral ground state in three-band superconductors," Journal of the Physical Society of Japan, vol. 79, Article ID 114706, 2010.

[49] Y. Tanaka and T. Yanagisawa, "Chiral state in three-gap superconductors," Solid State Communications, vol. 150, no. 41-42, pp. 1980-1982, 2010.

[50] T. Yanagisawa, Y. Tanaka, I. Hase, and K. Yamaji, "Vortices and chirality in multi-band superconductors," Journal of the Physical Society of Japan, vol. 81, no. 2, Article ID 024712, 2012.

[51] T. Yanagisawa and I. Hase, "Massless modes and Abelian Gauge fields in multi-band superconductors," Journal of the Physical Society of Japan, vol. 82, no. 12, Article ID 124704, 2013.

[52] Y. S. Yerin and A. N. Omelyanchouk, "Frustration phenomena in Josephson point contacts between single-band and threeband superconductors," Low Temperature Physics, vol. 40, no. 10, article 943, 2014.

[53] J. H. Kim and Z. Tešanović, "Effects of strong Coulomb correlations on the phonon-mediated superconductivity: a model inspired by Copper oxides," Physical Review Letters, vol. 71, no. 25, pp. 4218-4221, 1993.

[54] M. L. Kulić, "Importance of the electron-phonon interaction with the forward scattering peak for superconducting pairing in cuprates," Journal of Superconductivity and Novel Magnetism, vol. 19, no. 3, pp. 213-249, 2006.

[55] R. Szczęśniak, "Pairing mechanism for the high- $T_{C}$ superconductivity: symmetries and thermodynamic properties," PLoS ONE, vol. 7, no. 4, Article ID e31873, 2012.

[56] R. Szczesniak and A. P. Durajski, "Anisotropy of the gap parameter in the hole-doped cuprates," Superconductor Science and Technology, vol. 27, no. 12, Article ID 125004, 2014. 

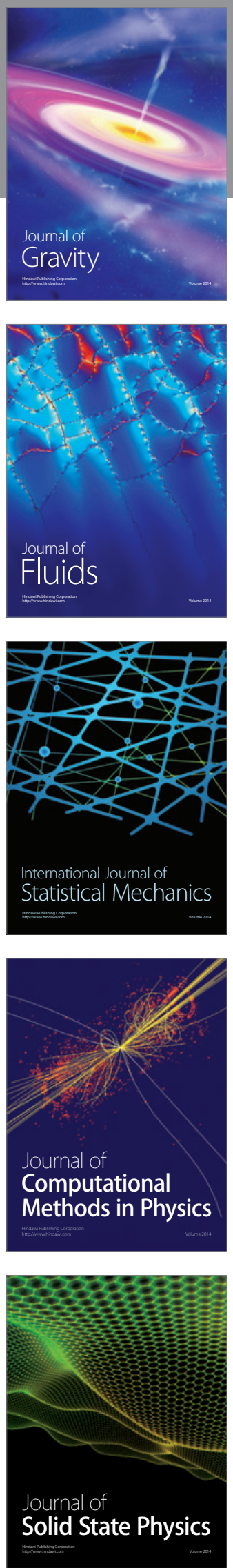

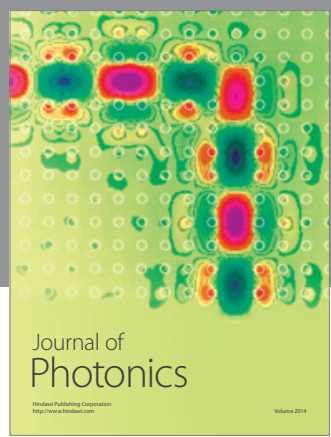

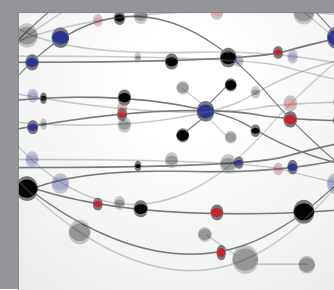

The Scientific World Journal

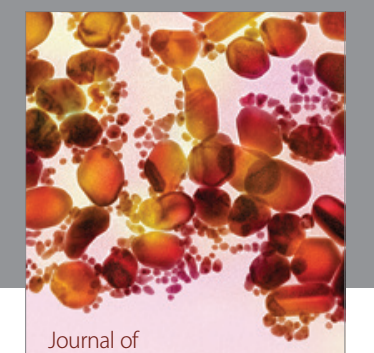

Soft Matter
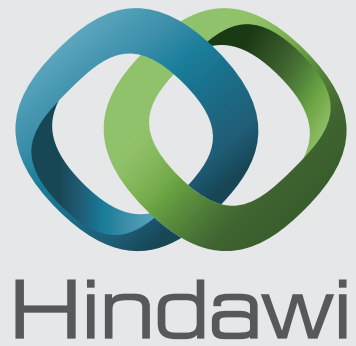

Submit your manuscripts at

http://www.hindawi.com
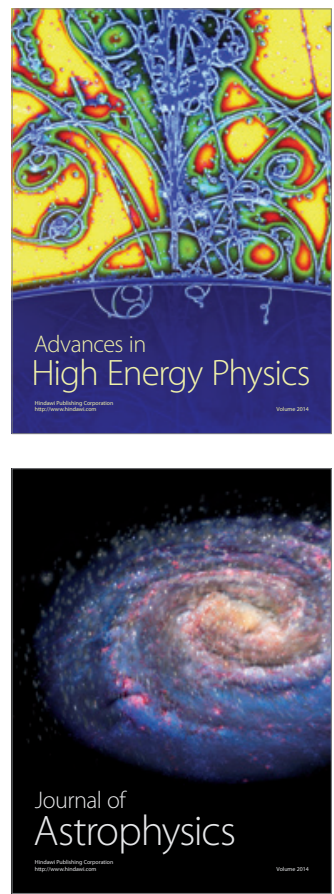
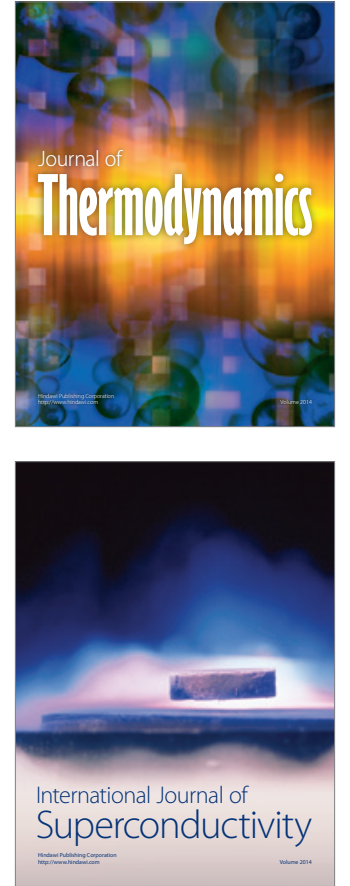
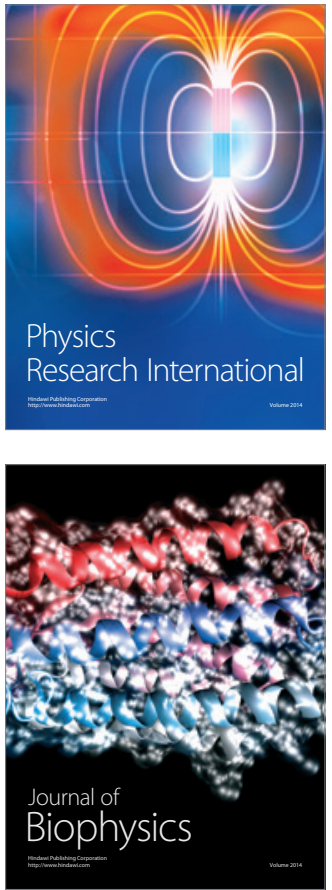
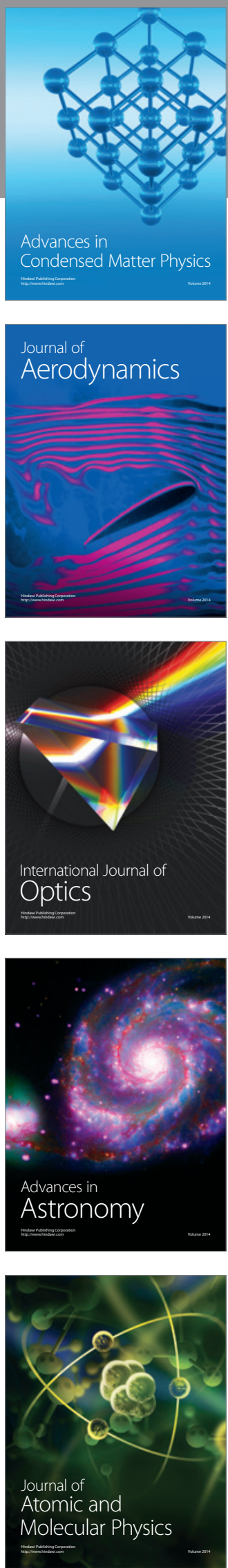